\section{ABSTRACT}

The primary purpose of this pilot study was to test the feasibility of an intervention designed to reduce care-resistant behaviors (CRBs) in persons with moderate-to-severe dementia during oral hygiene activities.

The intervention, Managing Oral Hygiene Using Threat Reduction (MOUTh), combined best oral hygiene practices with CRB reduction techniques. Oral health was operationalized as the total score obtained from the Oral Health Assessment Tool (OHAT). CRB was measured using a refinement of the Resistiveness to Care Scale. Seven nursing home residents with dementia received twice daily mouth care for 14 days. The baseline OHAT mean score of $7.29(S D=1.25)$ improved to 1.00 $(S D=1.26, p<.001)$; CRB improved from 2.43 CRBs/minute (SD $=4.26$ ) to $1.09 \mathrm{CRBs} /$ minute $(\mathrm{SD}=1.56, \mathrm{t}=1.97$, df $41, p=.06$ ).

The findings from this pilot study suggest that the MOUTh intervention is feasible and reduced CRBs, thus allowing more effective oral care.

KEY WORDS: oral hygiene,

dementia, nursing home residents,

care-resistant behaviors

\title{
An intervention to reduce care-resistant behavior in persons with dementia during oral hygiene: a pilot study
}

\author{
Rita A. Jablonski, PhD, CRNP; '* Barbara Therrien, PhD, RN, FAAN; ${ }^{2}$ \\ Ellen K. Mahoney, RN, DNS, FGSA; ${ }^{3}$ Ann Kolanowski, PhD, RN, FAAN; ${ }^{4}$ \\ Mia Gabello, BS, RN; ${ }^{5}$ Alexandra Brock, BS, $\mathrm{RN}^{5}$ \\ ${ }^{1}$ Assistant Professor, School of Nursing, Penn State University, University Park, Pennsylvania; ${ }^{2}$ Associate \\ Professor, University of Michigan School of Nursing, Ann Arbor, Michigan; ${ }^{3}$ Associate Professor, \\ Department Chair, William F. Connell School of Nursing, Boston College, Chestnut Hill, Massachusetts; \\ ${ }^{4}$ Elouise Ross Eberly Professor, School of Nursing, Penn State University, University Park, Pennsylvania; \\ ${ }^{5}$ Graduate Student, School of Nursing, Penn State University, Pennsylvania. \\ *Corresponding author e-mail: raj16@psu.edu
}

Spec Care Dentist 31(3): 77-87, 2011

\section{Introduction}

Throughout the past three decades, the oral health of nursing home $(\mathrm{NH})$ residents has worsened as a consequence of inadequate oral hygiene. ${ }^{1-4}$ The trend toward worsening oral health among NH residents is complicated by the rising numbers of persons entering NHs with some or all of their natural dentition; in fact, more than half of all NH residents are dentate. ${ }^{5}$ Resources necessary for maintaining oral health are limited in this population. Medicare does not cover routine dental care and Medicaid coverage varies among states but either does not cover dental care or compensates dentists so poorly that few will accept it. ${ }^{6}$ However, older adults, especially those with dementia, require meticulous daily oral hygiene.

Over half of all NH residents require assistance in all aspects of activities of daily living, including mouth care. ${ }^{7}$ One major barrier to the provision of that assistance is care-resistant behavior (CRB). CRBs are actions "invoked by a caregiving encounter, defined as the repertoire of behaviors with which persons with dementia withstand or oppose the efforts of a caregiver." ${ }^{8}$ In earlier research, behavior meeting this definition was usually labeled as "uncooperative behavior,"9-11 "disruptive behavior,"12-14 or even "agitation." 15 Volicer et al. ${ }^{15}$ and Mahoney et al. ${ }^{8}$ distinguished agitation from CRB using contextual cues: agitation usually occurs without a preceding event, while CRB occurs in response to a precipitating event. Agitation is evident in persons with mild dementia and increases with moderate dementia before waning as the dementia progresses to severe. ${ }^{15} \mathrm{CRB}$, however, increases exponentially as the severity of dementia increases; one study ${ }^{15}$ found an eightfold increase in overall CRB when dementia progressed to a severe stage. CRB is also associated with functional status: ${ }^{16}$ elders with dementia who require more assistance are more likely to exhibit CRB. The coexistence of cognitive impairments in persons requiring assistance with activities of daily living creates a clinical conundrum: elders who most need assistance with mouth care are most likely to resist helping behaviors.

In spite of the relationship between poor oral health and CRB during mouth 
care, researchers have focused their attention on providing educational interventions to nursing assistants and measuring oral health outcomes for $\mathrm{NH}$ residents without significant cognitive impairments or obvious CRB. For example, Pyle et al., ${ }^{17}$ Frenkel et al., ${ }^{3}$ Nicol et al., ${ }^{18}$ and Peltola et al. ${ }^{19}$ found that providing $\mathrm{NH}$ staff with intervention instruction led to clinically and statistically significant improvements in the oral health of older adults with dementia. None of the studies included persons with moderate-to-severe dementia and all excluded persons with any type of CRB. On the other hand, MacEntee et al. ${ }^{20}$ conducted a randomized clinical trial in $14 \mathrm{NHs}$ involving 113 elders and found that the educational intervention had no impact on either dental hygiene or oral health. Like the aforementioned studies, MacEntee's team excluded residents who could not or would not cooperate during the study. ${ }^{20}$

The primary purpose of this pilot study was to test the feasibility of an intervention designed to reduce CRB in persons with moderate-to-severe dementia during oral hygiene activities. The secondary aim was to determine if the reduction in CRB contributed to more effective mouth care, thus improving oral health. This unique intervention was based on the neurobiological principles of threat perception and fear response. When faced with a threat, all organisms react with fear-evoked "fight-or-flight" responses. These responses are both autonomic (e.g., elevated heart rate, sweating) and behavioral (e.g., escaping, attacking). Persons with dementia have heightened threat perception as a result of neurobiological changes that affect the cerebral cortex, hippocampus, and amygdala. ${ }^{21}$ These individuals may easily interpret mouth care, which is an intimate and potentially invasive procedure, as a threatening action by threatening people and may respond by exhibiting CRB.

The intervention we developed to reduce CRB during oral care is called Managing Oral Hygiene Using Threat Reduction (MOUTh), and includes (1) best oral hygiene practices for older adults, (2) early recognition of CRBs, and (3) implementation of a constella- tion of behavioral techniques designed to reduce threat perception and thereby prevent or de-escalate CRB.

\section{Theoretical foundation of MOUTh intervention}

The amygdala, in concert with the hippocampus and the cerebral cortex, normally perceives threat and initiates a rapid behavioral fear response, typically in the form of defensive, escape, or freezing actions. ${ }^{22,23}$ In persons with dementia, the deterioration of the brain, particularly the hippocampus and cortical structures, affects cognitive perception of potentially threatening stimuli and control of concomitant fear responses as degradation of the cortical-thalamic-amygdala pathway occurs. ${ }^{24}$ Thus, as dementia evolves, primitive threat identification and fear responses have little to no cortical control. As perception and reasoning become distorted, persons with dementia attribute high-threat to low-threat or nonthreatening situations. ${ }^{21,25}$

The MOUTh intervention contained two components: best mouth care practices for older adults with natural dentition and dentures, ${ }^{26-30}$ and strategies to reduce threat perception during the provision of mouth care. ${ }^{31-34}$ For example, best mouth care practices included using warm water for rinsing and using interdentate brushes for flossing. ${ }^{26-30}$ Threat-reduction strategies were a constellation of techniques congruent with neurobiological, dental, and nursing studies and are described in Table 1.

\section{Methods}

\section{Ethics}

The university Institutional Review

Board reviewed and approved this study. We recruited subjects from one 250-bed nonprofit NH located in central Pennsylvania. After consent was obtained from the residents' responsible parties, $\mathrm{NH}$ residents meeting initial criteria were screened for eligibility.

\section{Subjects}

NH staff identified NH residents who met initial criteria. These potential sub- jects had at least two adjacent teeth or consistently wore at least one removable denture, had a documented diagnosis of dementia or Alzheimer's disease, were aged 65 or older, and had a consistent history of care resistance during oral hygiene. Additional eligibility requirements included moderate dependence on others for care, moderate-to-severe dementia, and minimum mouth care ability. An advanced practice registered nurse screened subjects who met initial criteria using the Katz Activities of Daily Living instrument ${ }^{35}$ to determine dependence, the Global Deterioration Scale (GDS) to quantify dementia, ${ }^{36}$ and a range of motion activity to assess minimum mouth care ability. The participants were moderately dependent on others for activities of daily living, scoring a mean of 10.29 (SD = 3.25) on the Katz Activities of Daily Living instrument. ${ }^{35}$ The GDS instrument categorizes scores of 4 to 5 as moderate dementia and scores of 6 to 7 as severe dementia. The participants suffered from moderate-tosevere dementia, with a mean Global Deterioration Score ${ }^{36}$ of $5.86(S D=.90)$. Minimum mouth care ability was quantified as the ability of the NH resident to (1) grasp and hold a toothbrush or denture cup and (2) touch his or her mouth. Seven residents met all enrollment criteria. All were white; all but one were female. The mean age was 82.29 years $(\mathrm{SD}=4.31$ years $)$, and the mean time in the facility was 24.79 months ( $\mathrm{SD}=$ 15.49 years).

\section{Procedures}

All participants received a baseline oral health assessment by an advanced practice registered nurse using the Oral Health Assessment Tool (OHAT). Trained research staff then observed and documented CRBs during routine mouth care provided by the NH staff twice daily for 3 days, resulting in six preintervention data points. Mouth care occurred in residents' rooms.

Members of the research team provided mouth care according to the MOUTh intervention described above. All subjects were escorted to their rooms 


\section{Table 1. Threat-reduction strategies.}

Description of strategy

Approaching the resident at eye level and within his or her visual field ${ }^{34}$

Providing mouth care in a quiet environment with a minimum of persons present $^{34}$

Establishing rapport with the resident by engaging in affirming and simple conversation (e.g., complimenting a resident on his shirt) ${ }^{34}$

Using gentle touch judiciously ${ }^{31,34}$

Smiling when interacting with the resident ${ }^{34}$

Avoidance of "elderspeak," a term used to describe "baby talk" speech patterns associated with infants and pets but inappropriately employed when engaged with older adults: high pitch, short sentences, sing-song cadence, patronizing tone, use of collective pronouns, and infantilizing terms (baby, honey, dearie) 32,33

\section{Distraction $^{31}$}

Bridging: ${ }^{31}$ having the elder hold the same item being used in mouth care by the caregiver such as a toothbrush or denture cup. This technique is similar to distraction except the items are congruent with the care being provided.

Priming: ${ }^{31}$ using objects from the environment to help the elder to initiate or complete mouth care.

Chaining: ${ }^{31}$ the initiation of specific oral hygiene activities by the caregiver member with the expectation that the elder completes the activities.

Hand-over-hand: the placing of the caregiver's hands over the elder's hands and guiding the elder's hands. ${ }^{31}$ Hand-over-hand can be employed in conjunction with chaining.

Cueing: $:^{31,34}$ the use of polite, one-step commands. Cueing is the verbal analog of nonverbal priming.

Gestures and pantomiming ${ }^{34}$

Mirror-mirror: this technique was developed by the first author in her clinical practice. She found that persons with dementia who resisted care by not opening their mouths would open their mouths automatically if she placed them before a mirror and provided mouth care by standing BEHIND the elders and reaching around to brush and floss their teeth.

Rescuing: ${ }^{31}$ the replacement of one caregiver with another caregiver during any unsuccessful mouth care activity where CRBs are escalating
Rationale

Towering over a resident may invoke the threat response and CRBs.

Excessive noise and additional persons may cause the elder to interpret the situation as threatening.

Nonthreatening and personable behaviors on the part of the caregiver help to establish the situation as safe, and prevents the elder from assigning threat to a neutral encounter.

Gentle touch can reassure the elder and reduce anxiety

Research supports the relationship between the facial expressions of caregivers and the instigation of CRB in persons with dementia. Neutral, sad, angry, frustrated, and surprised faces were typically categorized as fearful; in contrast, only smiling faces were associated with happy emotional states. ${ }^{45,46}$ Relaxed and smiling caregivers were less likely to evoke CRBs. ${ }^{47}$

Elderspeak is a documented trigger to $\mathrm{CRB}^{32,33}$ because its dehumanizing approach heightens threat perception in persons with dementia.

Singing, talking, or providing a stuffed animal prevents or reduces CRBs. ${ }^{31}$ Bridging may access implicit memories, also described as procedural or unconscious memories, which are those memories surrounding specific tasks learned early in childhood and repeated throughout adult life. ${ }^{48,49}$ Although the elder is not actually performing his or her own mouth care, the elder may perceive that he or she is involved in mouth care and selfcare is unlikely to be perceived as threatening. ${ }^{47}$

Priming, such as bridging, also accesses implicit or procedural memories. ${ }^{48,49}$ The team members were taught to use priming by providing mouth care in front of a sink and placing toothbrushes in participants' hands instead of brushing their teeth. The rationale for this technique was that self-care is unlikely to be perceived as threatening. ${ }^{47}$

Chaining is used in conjunction with priming to encourage the elder to perform as much self-care as possible, because self-care is unlikely to be perceived as threatening by persons with dementia. ${ }^{47}$

Hand-over-hand reduces the perception of assault by the caregiver. ${ }^{31}$ We found this technique to be especially useful when removing dentures. The team member placed his or her hands over the resident's hands and guided the resident in the removal or insertion of dentures.

The ability of elders with dementia to process verbal communication, especially complex multistep directions, erodes with the progression of the disease. ${ }^{31,34}$ Cueing prevents verbal overload and subsequent threat perception.

The ability of elders with dementia to process verbal communication erodes with the progression of the disease. ${ }^{31,34} \mathrm{We}$ found gestures and pantomime to be important communication techniques that prevented and minimized frustration with caregivers and reduced threat perception.

We believe that "mirror-mirror" is another version of priming; its success may lay in the removal of the caregiver between the elder and his or her image in the mirror.

The escalation of CRBs during a mouth care session may be due to the perception of that particular caregiver as threatening. When the second caregiver replaces the first caregiver, the second one is perceived as a "rescuer" who saved the elder. The elder usually becomes more willing to engage in mouth care after being rescued by the safe second caregiver. ${ }^{31}$ 
or bathrooms (most rooms had sinks outside of the bathroom) for mouth care. The NH supplied soft toothbrushes and toothpaste. The research team provided plastic interdentate sticks and floss heads for flossing, as well as alcohol-free mouthwash for rinsing. Mouth care was provided twice daily for two full weeks: in the morning after breakfast (between 8:30 a.m. and 11 a.m.) and in the evening after supper (between 5:30 p.m. and 8:30 p.m.).

CRBs were measured during each mouth care session by a second member of the research team present in the room. The threat-reduction strategies that were initiated with all of the NH subjects from the onset of oral hygiene activities included the avoidance of elderspeak, and the use of cueing, gestures, and pantomimes. Additional threat-reduction strategies were used as needed by the individual elder as the oral hygiene activity progressed. Postintervention OHAT scores were obtained after the 14th mouth care session (evening of Intervention Day 7) and after the 28th mouth care session (evening of Intervention Day 14).

\section{Measures}

Oral health and CRBs were measured throughout the study.

Oral health was operationalized as the total score obtained from the OHAT. The OHAT was a modification of the Brief Oral Health Status Examination, ${ }^{37}$ an oral health instrument developed specifically for $\mathrm{NH}$ residents with moderate-to-severe dementia. Each of the OHATs eight categories was pertinent to specific oral structures and was scored from 0 (healthy) to 2 (unhealthy), resulting in scores ranging from 0 to 16 . The original authors of the instrument obtained internal consistency using test-retest percent agreements and intraand intercarer correlation coefficients for total scores. ${ }^{38}$ Intracarer total OHAT scores achieved a correlation coefficient of .78 $(p<.001)$; intercarer total OHAT scores achieved a correlation coefficient of .74 $(p<.001)$. Validity was determined by comparing each of the eight categories with accepted dental criteria and instruments using clinical examinations by a qualified dentist. ${ }^{38}$ As for reliability, Cronbach's alpha in this study was .793. The first author collected the initial OHAT scores prior to the initiation of the observation period. The fifth author collected the remaining OHAT scores.

$C R B$ was measured using a refinement of the Resistiveness to Care (RTC) Scale, which was developed specifically for use with persons with dementia. ${ }^{8}$ The original instrument, developed by Mahoney and colleagues, ${ }^{8}$ was comprised of 13 items, quantified according to duration and intensity (mild, moderate, or extreme) of each episodic CRB. Videotapes were used to capture CRBs. Each behavior was only measured once; the duration for all episodes was determined and was measured using time in categories $(0=$ none, 1 to 16 seconds $=1$; 17 to 59 seconds $=2 ; 1$ to 2 minutes $=$ 3 ; more than 2 minutes $=4$ ). CRB scores were obtained by multiplying the duration of each individual behavior $(0,1,2$, $3,4)$ with the most severe form of the observed behavior ( 1 for mild, 2 for moderate, 3 for extreme) and summing up the scores for a final CRB measurement. The original instrument was developed and tested using 68 subjects at three sites (311 observations), with $\alpha=.82$ and Kappa values ranging from .82 to $.92 .^{8}$

A limitation of the original RTC Scale was the inability to use it in its current form to measure CRBs in real time during a clinical encounter; up to now, the RTC was used to measure CRBs captured on videotape. With feedback from the instrument's developer, the first author modified the RTC. The refinements included removing the duration component, measuring the intensity for each individual behavior, counting the frequency of each behavior within the intensity category, summing all episodes of CRB, and then standardizing the scores by dividing the sum with the duration of mouth care (in minutes) to obtain the rate of CRBs. Another modification was the classification of toothbrush biting as "grab object." The original instrument did not address toothbrush biting but did contain the category "clench mouth." We characterized refusal to open the mouth as "clench mouth" and actual toothbrush biting as "grab object." These modifications resulted in a practical instrument that was used to effectively measure CRB during mouth care, the RTC-r. Interrater reliability of the RTC-r was obtained during the preintervention phase; team members who had less than $90 \%$ interrater reliability were retrained by the first author until interrater reliability reached 90\%. Reliability was measured using Cronbach's alpha, .72 .

\section{Results}

The primary purpose of this pilot study was to test the feasibility of an intervention designed to reduce CRBs in persons with moderate-to-severe dementia during oral hygiene activities. The secondary aim was to determine if the reduction in CRBs contributed to more effective mouth care, thus improving oral health. Data were analyzed using SPSS (version 17; Chicago, IL). Analyses included descriptive techniques, paired-sample $t$-tests, and one-way analysis of variance.

Mean baseline RTC rates for the six baseline observation points (staff providing mouth care) and the 28 intervention observation points (research team providing mouth care using MOUTh) are presented for each subject in Table 2. Also presented in Table 2 are the three OHAT scores (Baseline, Intervention Day 7, and Intervention Day 14) for each subject.

\section{Oral health}

The baseline OHAT mean score was 7.29 $(\mathrm{SD}=1.25)$. All of the subjects had dry, chapped lips and dry, fissured tongues. The majority had dry, red, swollen gums with minimal saliva present. Six had chunks of food and flecks of medication visible in all areas of their mouths. Two had food in two sections of their mouths. When the research team members initiated mouth care, they observed bleeding gums in all but one dentate subject. After 7 days of twice daily mouth care, the OHAT scores dramatically improved to $2.14(\mathrm{SD}=.90$, significance $<.001)$. The 
Table 2. Mean Resistance to Care (RTC) rates and total Oral Health Assessment Tool (OHAT) scores by subject.

\begin{tabular}{|l|c|c|c|c|c|} 
Subject & $\begin{array}{c}\text { Mean (SD) } \\
\text { baseline RTC } \\
\text { rate (behaviors/ } \\
\text { minute) }\end{array}$ & $\begin{array}{c}\text { Mean (SD) } \\
\text { intervention RTC } \\
\text { rate (behaviors/ } \\
\text { minute) }\end{array}$ & $\begin{array}{c}\text { OHAT } \\
\text { (baseline) }\end{array}$ & $\begin{array}{c}\text { OHAT (7 days } \\
\text { postintervention) }\end{array}$ & $\begin{array}{c}\text { OHAT (14 days } \\
\text { postintervention) }\end{array}$ \\
\hline 403 & $.95(2.00)$ & $.37(.89)$ & 8 & 1 & 0 \\
\hline 404 & $1.65(.63)$ & $.55(.47)$ & 8 & 2 & 2 \\
\hline 405 & $3.27(4.28)$ & $1.43(2.10)$ & 6 & 1 & $1 \mathrm{a}^{\mathrm{a}}$ \\
\hline 406 & $5.22(8.85)$ & $1.75(1.10)$ & 9 & 3 & 0 \\
\hline 407 & $1.00(.69)$ & $1.00(.86)$ & 6 & 3 & 0 \\
\hline 408 & $.38(.37)$ & $.25(.52)$ & 6 & 3 & $3^{\mathrm{b}}$ \\
\hline 409 & $4.58(4.17)$ & $2.53(2.47)$ & 8 & 2 & 0 \\
\hline
\end{tabular}

aUnable to obtain OHAT score.

${ }^{b} \mathrm{New}$ complaint of mouth pain.

areas of most improvement were the cleanliness and moistness of the oral cavity and its structures. Gum bleeding ceased for all subjects. After 14 days of twice daily mouth care, the OHAT scores modestly improved to $1.00(\mathrm{SD}=1.26)$. The significance between the baseline
OHAT mean and the final OHAT mean was $p<.001$; between Day 7 and Day $14, p=.23$. Two of the individual components of the OHAT, condition of natural dentition and condition of dentures, contained conditions that were not amenable to the efforts of the research team: broken teeth and plaque on dentures. These conditions were reflected in the final OHAT scores for subjects 404 and 409. All but one subject showed progressive improvement during the 14-day intervention period; subject 409 complained of mouth pain during the last OHAT examination, which increased her score.

\section{Care-Resistant Behaviors}

Figures 1 through 7 illustrate the CRB rate for each data collection point for each individual subject. The most frequent CRBs were grabbing the caregiver (127 occurrences), saying no (126 occurrences), turning away (114 occurrences), grabbing objects, which included biting down on the toothbrush (59 occurrences), and pushing away the caregiver (35 occurrences). The mean CRB rate during the 3-day baseline observation period was 2.43 behaviors/minute $(\mathrm{SD}=$ 4.26). The mean CRB rate during the 14-day intervention period decreased to $1.09 \mathrm{CRBs} /$ minute $(\mathrm{SD}=1.56)$ but was not statistically significant $(\mathrm{t}=1.97$ [df 41, $p=.06]$ ).

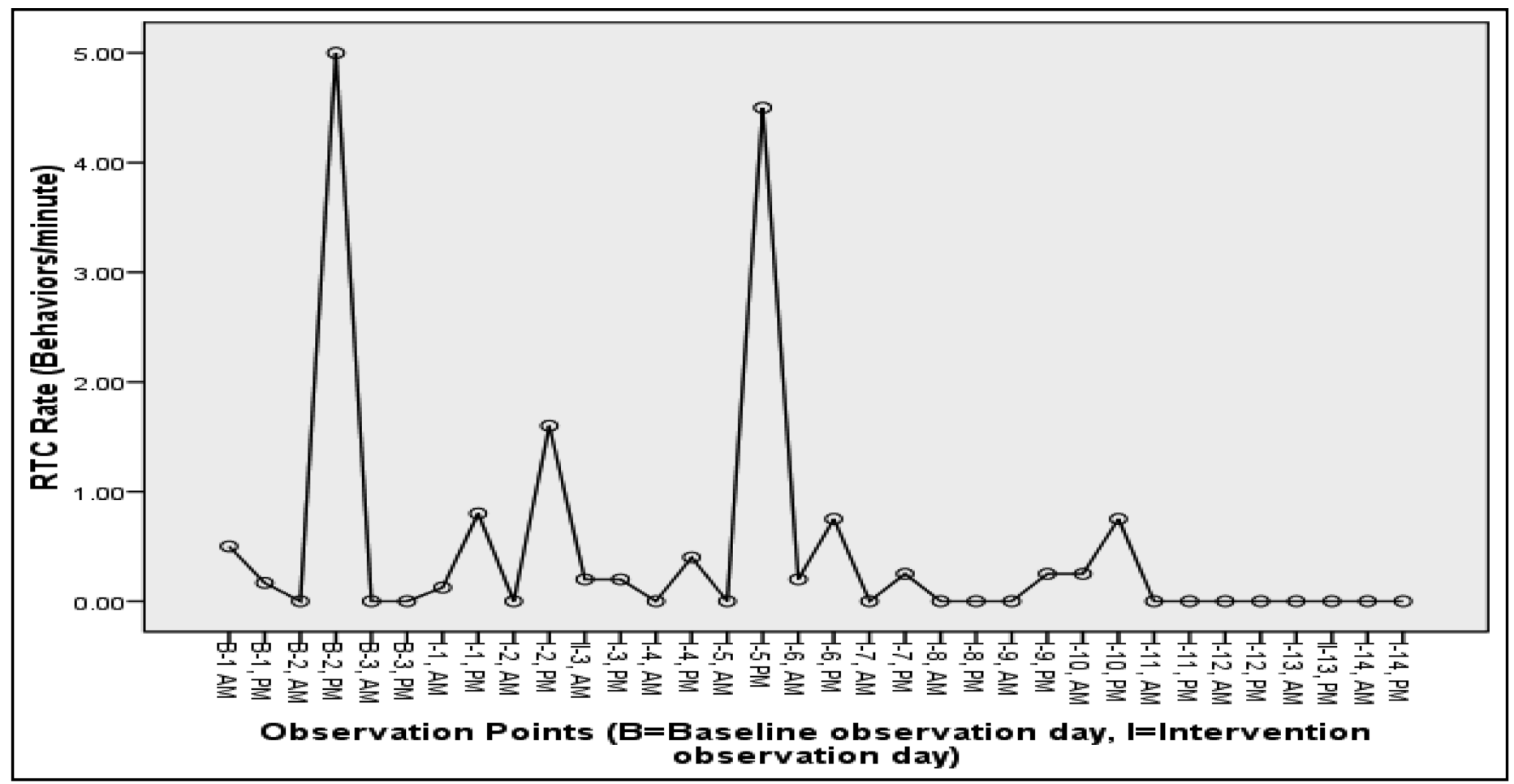

Figure 1. Subject 403 graph of RTC rates by observation points. 


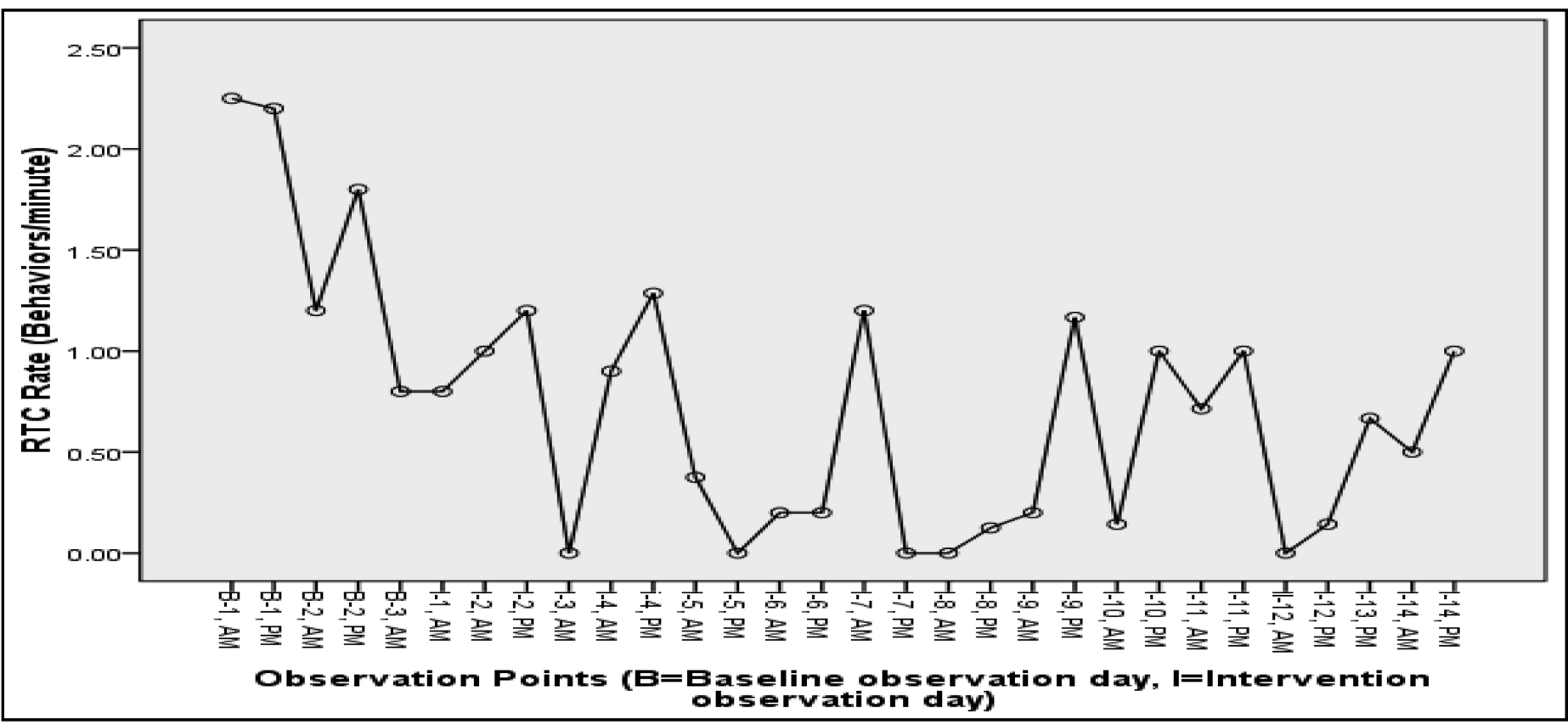

Figure 2. Subject 404 graph of RTC rate by observation points.

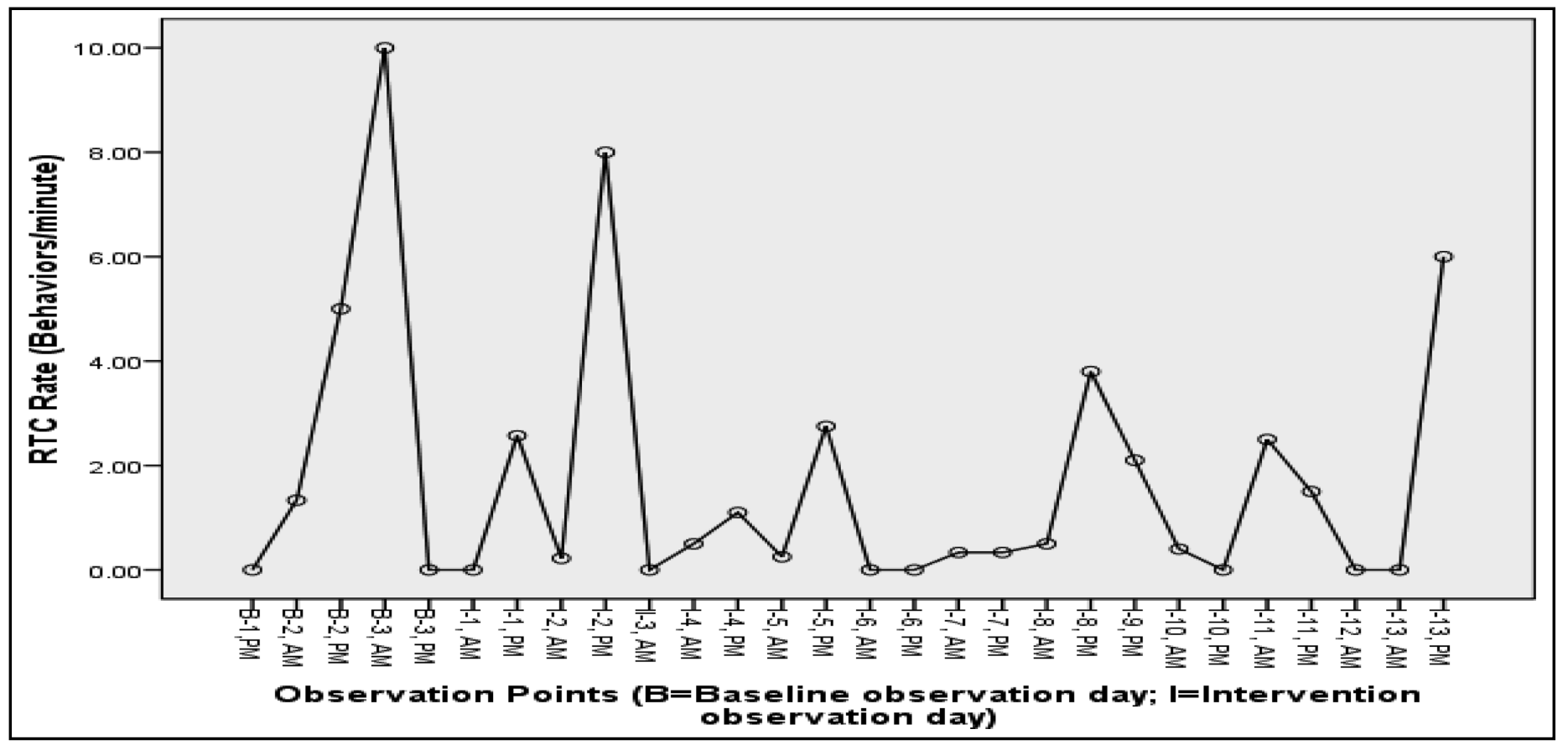

Figure 3. Subject 405 graph of RTC by observation points.

As noted earlier, the threat-reduction strategies that were employed universally with all of the subjects included elderspeak avoidance, cueing, gestures, bridging, and pantomimes. The remaining threat-reduction strategies were individu- ally applied, depending on the individual preferences of the subject. For example, one subject required the distraction of singing throughout the provision of oral care. In fact, if the research team member sang the directions (e.g., sang "spit"), the subject would respond to the command. All but one of the subjects preferred interdentate sticks over floss heads for flossing.

As illustrated by the graphs in Figure 1 through Figure 7, CRB rates did trend 


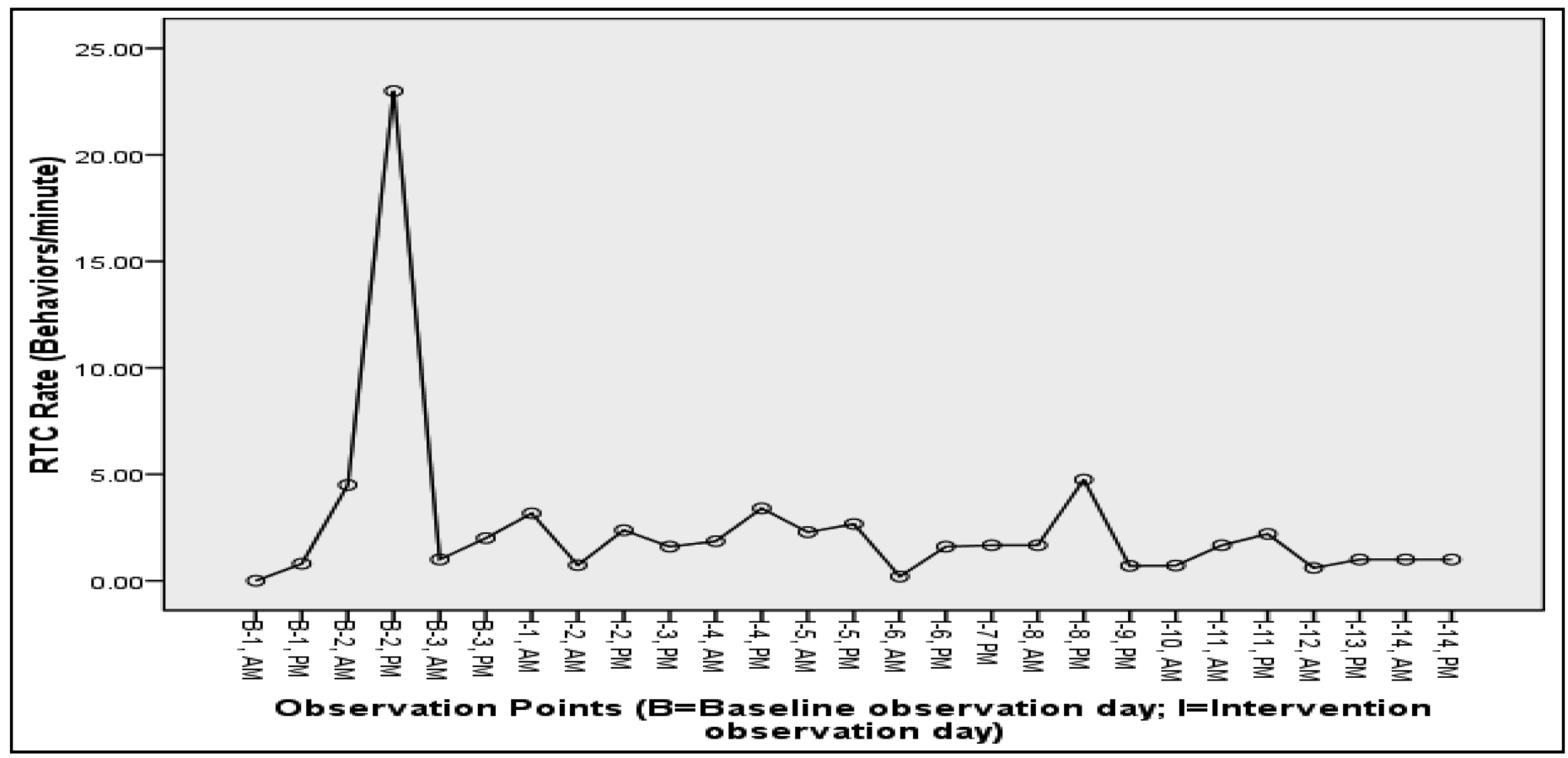

Figure 4. Subject 406 graph of RTC by observation points.

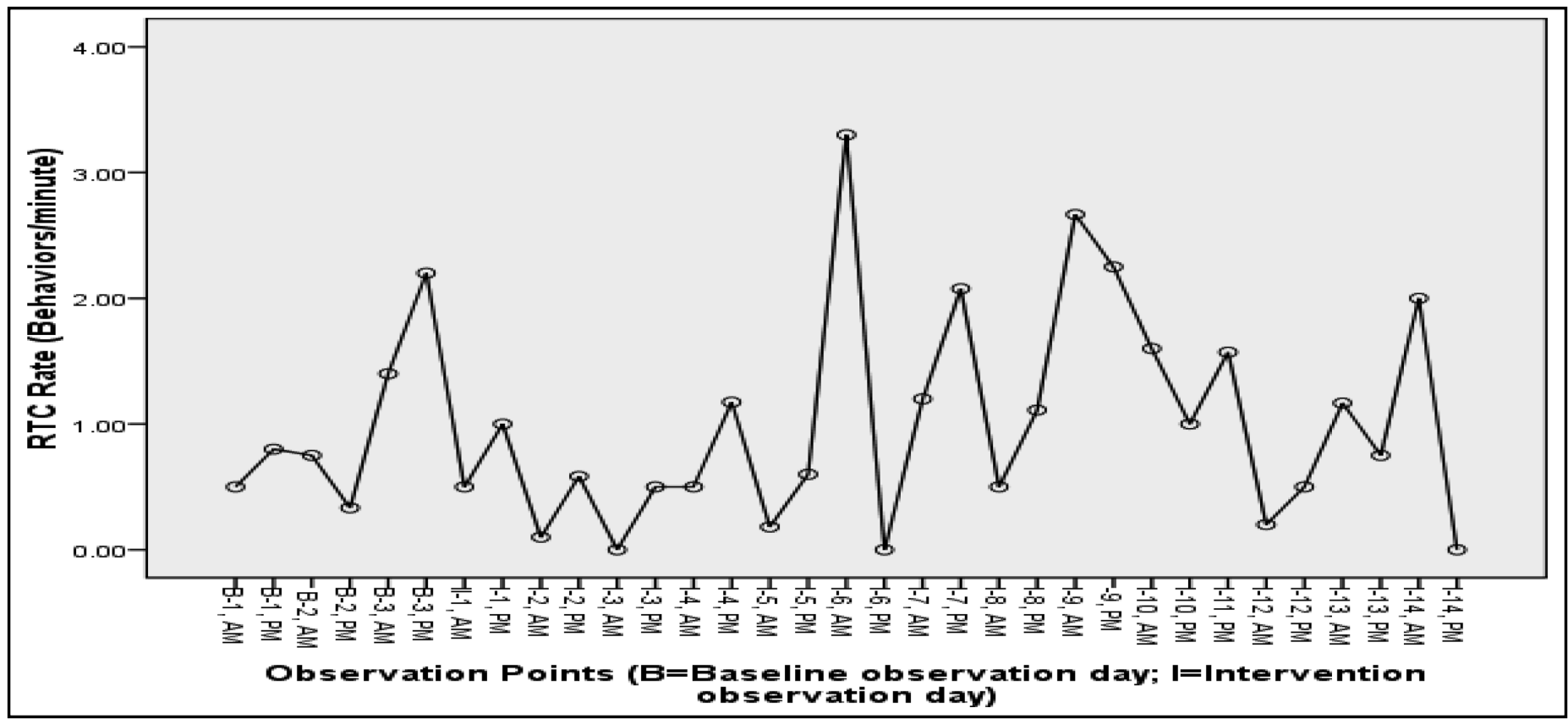

Figure 5. Subject 407 graph of RTC behaviors by observation points.

downward during the intervention phase, but there was a great deal of variability within and between subjects. Data that may have explained variability, such as acute infections or medication changes, were not collected.

\section{Discussion}

This pilot study proved informative and the lessons learned have informed the design of a proposed randomized clinical trial. As noted in the results section, some threat-reduction strategies were used with all of the subjects and were well received: elderspeak avoidance, cueing, gestures, bridging, and pantomimes. The use of other threat-reduction strategies depended on the individual subject; one subject's preferred threat-reduction strategy was 


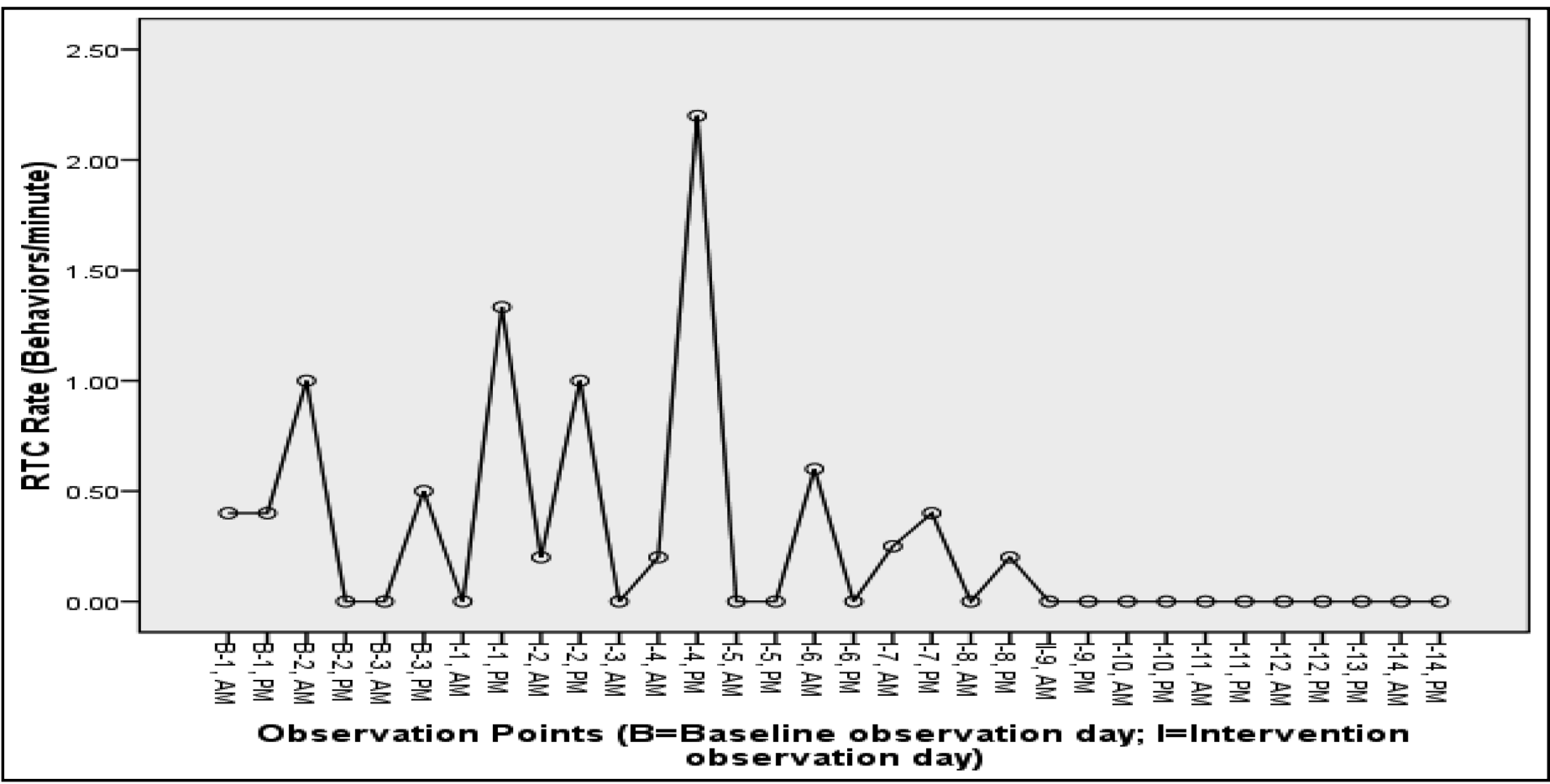

Figure 6. Subject 408 graph of RTC by observation points.

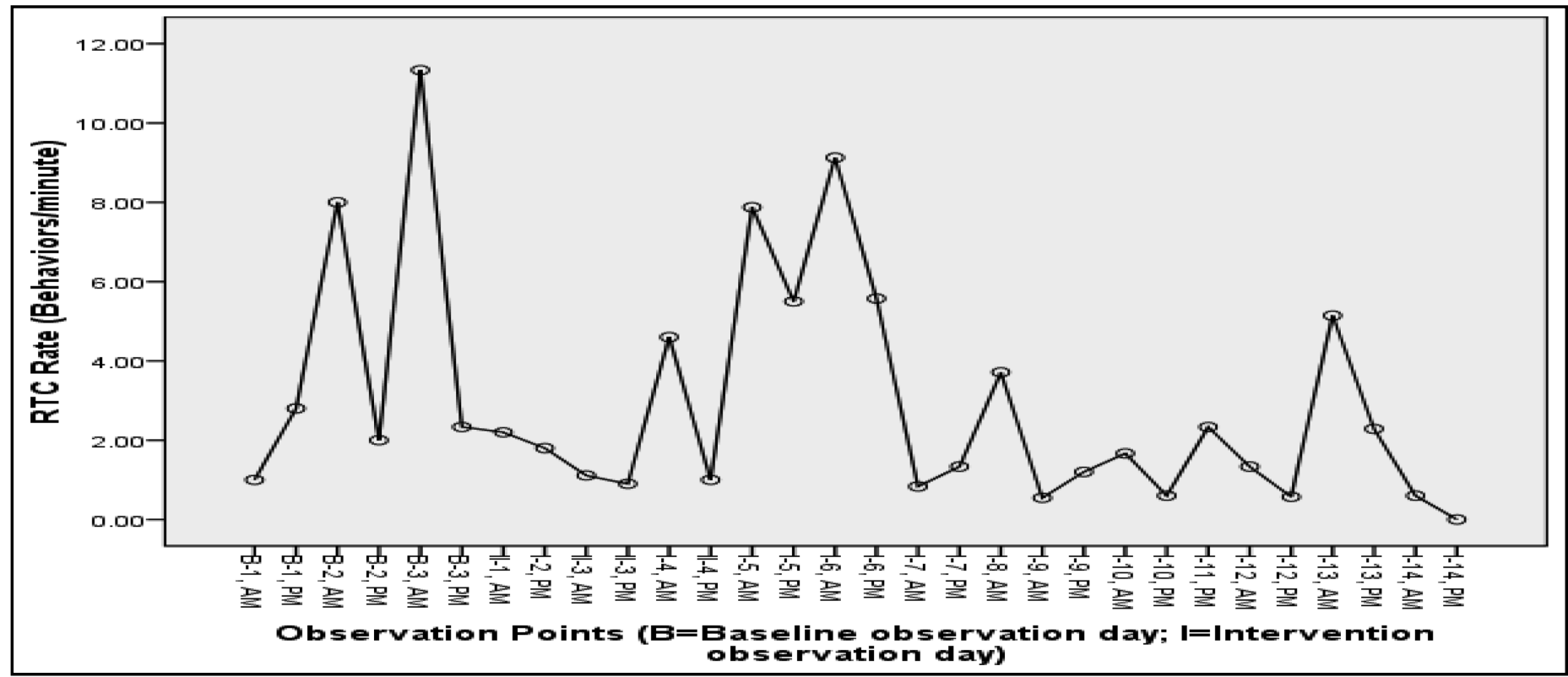

Figure 7. Subject 409 graph of RTC by observation points.

another's antecedent to CRB. For example, distraction in the form of singing was effective with one subject but was absolutely not tolerated by three other subjects. We determined that techniques would be effective through trial and error.
Another important lesson learned pertained to setting up the rooms with mouth hygiene supplies prior to entering the room with the subject. Initially, research team members brought the subject to his or her room and then produced the supplies. The rationale for this decision was to prevent other residents from removing the unmonitored supplies. On the first day of the intervention period, we discovered that CRBs started as soon as we began organizing 
supplies in front of the subject. On the second day, we organized the supplies immediately prior to taking the subject to his or her room. If CRBs occurred, they occurred during the mouth care interaction, not before. One possible explanation may be that altering the environment prior to the arrival of the subject helped reduce threat perception by cueing the subject to the purpose of the interaction.

The person observing the mouth care and recording CRBs did not always see the subject bite the toothbrush or clench his or her mouth. To improve the accuracy of the CRB reporting, the person providing the mouth care communicated episodes of toothbrush biting and mouth clenching to the person recording the CRBs. Two subjects repeatedly sucked on the toothbrushes; these behaviors were not recorded as CRBs.

The findings from this pilot study suggest that the MOUTh intervention helped to reduce CRBs by NH residents with moderate-to-severe dementia, thus allowing members of the research team to provide effective mouth care. The frequency and type of CRBs noted in this study were similar to those reported by other researchers. ${ }^{39}$ CRBs are a common problem encountered by nursing assistants during the provision of mouth care; $80 \%$ of nursing assistants have experienced CRBs. ${ }^{40}$ The number of CRBs associated with mouth care provided by nursing assistants during the observation period, however, may have been artificially reduced because mouth care was divorced from usual morning and evening care. During day shifts, the usual practice of nursing assistants is to integrate mouth care with total morning care: NH elders may have their teeth brushed as they sit on a commode or are suspended midair in a lift device, as documented by Coleman and Watson. ${ }^{41}$ During the observation period of this study, nursing assistants provided mouth care outside of usual morning care, and subjects were walked to their own rooms and placed before a sink. By providing mouth care in this fashion, the nursing assistants may have inadvertently been tapping into implicit memory, which may have decreased the number of CRBs that would normally have occurred. The nursing assistants in Coleman and Watson's study ${ }^{41}$ did not know that the focus of the study was on oral care; they were told that the purpose of the study was to observe overall morning care routines. Had the study been designed slightly differently, we may have recorded higher CRBs during the observation period.

When the nursing assistants provided mouth care during the observation period, subjects' teeth were rapidly and haphazardly brushed, no flossing occurred, and dentures were brushed in the older adults' mouths rather than being removed. The nursing assistants were more likely to do the mouth care for the older adult instead of allowing the elder to do as much care as possible. These practices may have accounted for the higher CRBs observed during the observation period; other researchers have demonstrated that CRBs during mouth care were most likely to occur when nursing assistants provided physical assistance rather than cueing the $\mathrm{NH}$ resident or allowing him/her to perform his or her own mouth care. ${ }^{41}$ Research team members, on the other hand, were taught to encourage the elder to perform as much mouth care as possible. These findings from this study support the theoretical foundation of the MOUTh intervention: CRB is a fear-evoked response to caregivers' unintentionally threatening behavior during mouth care. Thus, by reducing threat perception and fear response during mouth care, the research team members were able to provide more comprehensive mouth care.

These findings also suggest a relationship between CRB and poor oral hygiene in NH residents with dementia. Some study findings have supported this relationship, although this was not their original intent. Adam and Preston ${ }^{42}$ analyzed the effect of dementia on oral health and found that persons with moderate-to-severe dementia had twice as much dental plaque as persons with mild or no dementia, although this finding did not reach statistical significance. Adam and Preston, however, excluded persons who resisted mouth care. ${ }^{42}$ In a descriptive study designed to assess the oral care needs of NH residents, researchers concluded $^{43}$ that dental hygiene was inadequate for $60 \%$ of the 321 residents in two facilities. When the researchers separated the residents into "cooperative" and "uncooperative" groups, they found that $90 \%$ of the cooperative residents had adequate oral hygiene, while only $10 \%$ of the uncooperative residents did. Similarly, Samson et al. ${ }^{44}$ evaluated the impact of their educational program on the oral health of $\mathrm{NH}$ residents in one facility for 6 years. They found sustained improvement in oral hygiene for $70 \%$ of the residents; the $30 \%$ who did not demonstrate improvement included persons with dementia who resisted care. ${ }^{44}$

Another significant contribution of this study was the demonstration that flossing could be safely done in this population without automatically increasing CRBs. Flossing was accomplished using interdentate sticks, which look like plastic toothpicks with little spiral

"Christmas trees" on the end. The use of interdentate sticks was well tolerated by all of the subjects, save one who preferred the floss head. The use of interdentate sticks did not require subjects to open their mouths. Some of the subjects actually appeared to enjoy the interdentate sticks.

Initially, implementation of the MOUTh intervention was time-consuming: 9.5 minutes on average. As the research team members became more familiar with the individual residents, and more skilled with the MOUTh intervention, the duration of mouth care decreased to 4.83 minutes. While not statistically significant, there is a huge clinical difference between a 5-minute and a 10-minute activity. It may be difficult to convince NH staff to adopt our intervention if it proves to require more time than usual care practices. On the other hand, it is much more pleasurable for both the NH resident and the caregiver to have an enjoyable interaction during the provision of oral hygiene than to literally engage in a mouth care battle. NH staff may be willing to exchange a lengthier but positive interaction for a 
brief but unsatisfactory and unpleasant one.

Limitations of this study were the small sample size of seven subjects and the lack of ethnic diversity. This pilot study, however, did demonstrate the feasibility of the MOUTh intervention and produced promising results. We anticipate additional testing of the MOUTh intervention with larger and more diverse samples. We also plan to examine other variables that may contribute to changes in CRBs, such as delirium resulting from acute infections or pharmacological side effects. We plan to test this intervention in multiple sites using a randomized repeated measures design.

\section{Conclusion}

We demonstrated that the MOUTh intervention was feasible for $\mathrm{NH}$ residents with moderate-to-severe dementia. The intervention helped to reduce CRB occurring during mouth care, thus allowing the research team members to provide twice daily mouth care. The twice daily mouth care improved the oral health of these elders. We approached $\mathrm{CRB}$ as a physiological response to threat, rather than a "normal" component of dementia. We plan to continue to test the MOUTh intervention using larger and more diverse sample sizes.

\section{Acknowledgements}

The Brookdale Leadership in Aging Foundation provided support and funding for this pilot study.

\section{References}

1. Bagramian RA, Heller RP. Dental health assessment of a population of nursing home residents. J Gerontol 1977;32:168-74.

2. Kiyak HA, Grayston MN, Crinean CL. Oral health problems and needs of nursing home residents. Community Dent Oral Epidemiol 1993;21:49-52.

3. Frenkel H, Harvey I, Newcombe RG. Improving oral health in institutionalised elderly people by educating caregivers: a randomised controlled trial. Community Dent Oral Epidemiol 2001;29:289-97.
4. Murray PE, Ede-Nichols D, Garcia-Godoy F. Oral health in Florida nursing homes. Int $\mathrm{J}$ Dent Hyg 2006;4:198-203.

5. Dey AN. Characteristics of elderly nursing home residents: data from the 1995 National Nursing Home Survey. Adv Data 1997;289:1-8.

6. Jablonski RA, Munro CL, Grap MJ, Elswick RK. The role of biobehavioral, environmental, and social forces on oral health disparities in frail and functionally dependent nursing home elders. Biol Res Nurs 2005;7:75-82.

7. Jones AL, Dwyer LL, Bercovitz AR, Strahan GW. The National Nursing Home Survey: 2004 overview. Vital Health Stat 2009;13;1-155.

8. Mahoney EK, Hurley AC, Volicer L, et al. Development and testing of the Resistiveness to Care Scale. Res Nurs Health 1999;22:27-38.

9. Kambhu PP, Levy SM. Oral hygiene care levels in Iowa intermediate care facilities. Spec Care Dentist 1993;13:209-14.

10. Adams R. Qualified nurses lack adequate knowledge related to oral health, resulting in inadequate oral care of patients on medical wards. J Adv Nurs 1996;24:552-60.

11. Pyle MA, Jasinevicius TR, Sawyer DR, Madsen J. Nursing home executive directors' perception of oral care in long-term care facilities. Spec Care Dentist 2005;25:111-7.

12. Algase DL, Beck C, Kolanowski A, et al. Need-driven dementia-compromised behavior: an alternative view of disruptive behavior. Am J Alzheimer's Dis Other Demen 1996;11:10-9.

13. Kolanowski AM, Whall AL. Toward holistic theory-based intervention for dementia behavior. Holist Nurs Pract 2000;14:67-76.

14. Whall A. Developing needed interventions from the need-driven dementia-compromised behavior model. J Gerontol Nurs 2002;28:5.

15. Volicer L, Bass EA, Luther SL. Agitation and Resistiveness to Care are two separate behavioral syndromes of dementia. J Am Med Dir Assoc 2007;8:527-32.

16. Beck C, Heacock P, Mercer SO, Walls RC, Rapp CG, Vogelpohl TS. Improving dressing behavior in cognitively impaired nursing home residents. Nurs Res 1997;46:126-32.

17. Pyle MA, Massie M, Nelson S. A pilot study on improving oral care in long-term care settings. Part II: procedures and outcomes. J Gerontol Nurs 1998;24:35-8.
18. Nicol R, Petrina Sweeney M, McHugh S, Bagg J. Effectiveness of health care worker training on the oral health of elderly residents of nursing homes. Community Dent Oral Epidemiol 2005;33:115-24.

19. Peltola P, Vehkalahti MM, Simoila R. Effects of 11-month interventions on oral cleanliness among the long-term hospitalised elderly. Gerodontology 2007;24:14-21.

20. MacEntee MI, Wyatt CC, Beattie BL, et al. Provision of mouth-care in long-term care facilities: an educational trial. Community Dent Oral Epidemiol 2007;35:25-34.

21. Henry JD, Thompson C, Ruffman T, et al. Threat perception in mild cognitive impairment and early dementia. J Gerontol B Psychol Sci Soc Sci 2009;64:603-7.

22. LeDoux J. The emotional brain, fear, and the amygdala. Cell Mol Neurobiol 2003;23:727-38.

23. Maren S. Building and burying fear memories in the brain. Neuroscientist 2005;11:89-99.

24. LaBar KS, Torpey DC, Cook CA, et al. Emotional enhancement of perceptual priming is preserved in aging and early-stage Alzheimer's disease. Neuropsycologia 2005;43:1824-37.

25. Corcoran KA, Desmond TJ, Frey KA, Maren $S$. Hippocampal inactivation disrupts the acquisition and contextual encoding of fear extinction. J Neurosci 2005;25:8978-87.

26. American Dental Association. Oral Health Topics: Dentures; 2010. Available at: http://www.ada.org/2996.aspx [Accessed March 13, 2010].

27. American Dental Association. Oral Health Topics: Cleaning Your Teeth \& Gums. Available at: http://www.ada.org/2624.aspx [Accessed March 13, 2010].

28. Gil-Montoya JA, de Mello AL, Cardenas CB, Lopez IG. Oral health protocol for the dependent institutionalized elderly. Geriatr Nurs 2006;27:95-101.

29. Chalmers J, Pearson A. Oral hygiene care for residents with dementia: a literature review. J Adv Nurs 2005;52:410-9.

30. Chalmers J, Johnson V, Tang JH, Titler MG Evidence-based protocol: oral hygiene care for functionally dependent and cognitively impaired older adults. J Gerontol Nurs 2004;30:5-12.

31. Chalmers JM. Behavior management and communication strategies for dental 
professionals when caring for patients with dementia. Spec Care Dent 2000;20:147-54.

32. Williams KN, Herman R, Gajewski B, Wilson K. Elderspeak communication: impact on dementia care. Am J Alzheimers Dis Other Demen 2009;24:11-20.

33. Herman RE, Williams KN. Elderspeak's influence on resistiveness to care: focus on behavioral events. Am J Alzheimers Dis Other Demen 2009;24:417-23.

34. Kayser-Jones J, Bird WF, Redford M, Schell ES, Einhorn SH. Strategies for conducting dental examinations among cognitively impaired nursing home residents. Spec Care Dentist 1996;16:46-52.

35. Katz S, Ford AB, Moskowitz RW, Jackson BA, Jaffe MW. Studies of illness in the aged: the index of ADL: a standardized measure of biological and psychosocial function. JAMA 1963;185:914-9.

36. Reisberg B, Ferris SH, de Leon M, Crook T. The Global Deterioration Scale for assessment of primary degenerative dementia. Am J Psychiatry 1982;139:1136-9.

37. Kayser-Jones J, Bird WF, Paul SM, Long L, Schell ES. An instrument to assess the oral health status of nursing home residents. Gerontologist 1995;35:814-24.

38. Chalmers JM, King PL, Spencer AJ, Wright FA, Carter KD. The oral health assessment tool-validity and reliability. Aust Dent J 2005;50:191-9.

39. Chalmers JM, Levy SM, Buckwalter KC, Ettinger RL, Kambhu PP. Factors influencing nurses' aides' provision of oral care for nursing facility residents. Spec Care Dent 1996;16:71-9.
40. Frenkel HF. Behind the screens: care staff observations on delivery of oral health care in nursing homes. Gerodontology 1999;16:75-80.

41. Coleman P, Watson NM. Oral care provided by certified nursing assistants in nursing homes. J Am Geriatr Soc 2006;54:138-43.

42. Adam H, Preston AJ. The oral health of individuals with dementia in nursing homes. Gerodontology 2006;23:99-105.

43. Montal S, Tramini P, Triay JA, Valcarcel J. Oral hygiene and the need for treatment of the dependent institutionalised elderly. Gerodontology 2006;23:67-72.

44. Samson H, Berven L, Strand GV. Long-term effect of an oral healthcare programme on oral hygiene in a nursing home. Eur J Oral Sci 2009;117:575-9. 